\title{
Unraveling lipid/protein interaction in model lipid bilayers by Atomic Force Microscopy ${ }^{\dagger}$
}

\author{
Andrea Alessandrini ${ }^{a, b}$ and Paolo Facci ${ }^{a *}$
}

\begin{abstract}
The current view of the biological membrane is that in which lipids and proteins mutually interact to accomplish membrane functions. The lateral heterogeneity of the lipid bilayer can induce partitioning of membrane-associated proteins, favoring protein-protein interaction and influence signaling and trafficking. The Atomic Force Microscope allows to study the localization of membrane-associated proteins with respect to the lipid organization at the single molecule level and without the need for fluorescence staining. These features make AFM a technique of choice to study lipid/protein interactions in model systems or native membranes. Here we will review the technical aspects inherent to and the main results obtained by AFM in the study of protein partitioning in lipid domains concentrating in particular on GPI-anchored proteins, lipidated proteins, and transmembrane proteins. Whenever possible, we will also discuss the functional consequences of what has been imaged by Atomic Force Microscopy. Copyright $\subset 2011$ John Wiley \& Sons, Ltd.
\end{abstract}

Keywords: protein-lipid interaction in model membranes; AFM; SLBs; transmembrane proteins

\section{INTRODUCTION}

In the Singer and Nicholson model (Singer and Nicholson, 1972) of the biological membrane the lipids were considered mainly as a solvent for the membrane proteins allowing them to diffuse in order to interact with each other and perform their functional activity. It has become clear now that lateral heterogeneity is the main aspect of the lipid bilayer structure and that the lateral organization of the membrane affects many membrane functions such as signal transduction, protein and lipid sorting, and endocytosis (Sackmann, 1984; Engelman, 2005). The first evidences for the presence of laterally organized domains in the lipid bilayer came from the study of model systems. Later on, it was hypothesized that also biological membranes were characterized by lateral heterogeneity as suggested by the presence of detergent resistant domains (DRM). This experimental observation led to the introduction of the term 'raft' to designate globally the presence of specialized domains of lipids and proteins in the lateral organization of lipid bilayers (Simons and Ikonen, 1997; Jacobson et al., 2007). The identification of these raft domains has remained elusive in biological membranes, at variance with model systems, leading to the conclusion that these functional domains, if they exist, must be small (in the nanometer range) and transient (characterized by a short aggregation lifetime for the constituent molecules) (Lingwood and Simons, 2010). Hence, the biological structures associated with the definition of 'rafts' are highly dynamic structures in the nanometer range. It has recently become possible, with the introduction of super-resolution microscopy, to obtain strong evidences of the existence of transient domains also in the membrane of living cells (Eggeling et al., 2009). Moreover, it has been demonstrated that many membrane proteins require the presence of specific lipids for proper function (Lee, 2009). For example, anionic lipids are necessary for the functionality of certain ion channels, probably through a binding to positively charged residues (Schmidt et al., 2006; Marius et al., 2008). The up-to-date view of the biological membrane is that in which lipids and proteins strongly influence each other by fine mechanisms involving chemical and physical interactions (Jensen and Mouritsen, 2004; Lee, 2005).

In a reductionist approach it is possible to exploit model systems of the complex biological membranes to retrieve information about the physical rules, which regulate the phase behavior of the membranes and the interplay between protein and lipids. Three model systems are mainly used to accomplish this task: Giant Unilamellar Vesicles (GUV) in which membrane proteins have been reconstituted; Black Lipid Membranes (BLM), which are useful for the studies of functional properties of transport proteins; supported lipid bilayers (SLB) with reconstituted membrane proteins. It is clear that an approach based on the exploitation of various model systems and different investigation techniques offers a better understanding of the complex lipid/protein interactions which might be relevant to accomplish membrane functions. The SLB model system offers the great advantage of being suitable to be studied with many surface sensitive techniques. Among these techniques, of particular relevance is the Atomic Force Microscopy (AFM) (Mingeot-Leclercq et al., 2008), which provides high lateral and vertical space resolution allowing the study of the presence of different domains in the bilayer and the distribution of

* Correspondence to: P. Facci, CNR-Istituto Nanoscienze, Via Campi 213/A, 41125 Modena, Italy.

E-mail: paolo.facci@unimore.it

a A. Alessandrini, P. Facci

CNR-Istituto Nanoscienze, Via Campi 213/A, 41125 Modena, Italy

b A. Alessandrini

Department of Physics, University of Modena and Reggio Emilia, Via Campi 213/A, 41125 Modena, Italy

This article is published in Journal of Molecular Recognition as a focus on AFM on Life Sciences and Medicine, edited by Jean-Luc Pellequer and Pierre Parot (CEA Marcoule, Life Science Division, Bagnols sur Cèze, France). 
membrane proteins relative to the domains. This capability is connected to the fact that the coexistence of different lipid phases can be detected by AFM due to the height difference among the phases, which is related to the degree of chain order.

In this work we will review what the AFM technique can offer in the study of lipid-protein interactions. The research field of lipid-protein interactions includes the relations between transmembrane proteins and lipids, the interactions of a lipid bilayer with surface bound proteins, lipoproteins, and peptides. In this work we will mainly concentrate on lipoproteins and transmembrane proteins highlighting the information retrieved by AFM on these systems in comparison with other techniques on different model systems. The focus will be on the distribution of membrane proteins relative to phase separation of the bilayer. AFM has also provided a number of information on the structure of the surface-exposed portion of transmembrane proteins and on their oligomeric structure. Impressive results have been obtained in this field establishing the AFM technique as the only technique which can obtain subnanometer lateral resolution on membrane proteins in physiologic-like environment. Moreover, the possibility of observing the proteins at work in the real space is another extremely useful advantage of AFM (Engel and Müller, 2000). Several reviews about this topic can be found in the literature and the interested reader is referred to these papers (Müller et al., 2002; Engel and Gaub, 2008). Moreover, AFM applied in the force spectroscopy mode can provide a wealth of information on protein/lipid interactions (Müller, 2008). Experiments in which single membrane proteins are extracted from the lipid bilayer while measuring the opposing forces by AFM will not be reviewed here. The interested readers can find excellent reviews on this topic in the literature (Müller and Engel, 2007).

This review is organized as follows. In Chapter 1, the model system exploited in AFM studies will be critically described along with techniques to prepare SLBs with reconstituted membrane proteins on different surfaces. Chapter 2 will present results from the literature on the study of membrane protein-lipid interactions. In cases where information is available we will also relate the data obtained from AFM studies to the functional behavior of membrane proteins. In Chapter 3, we will describe the technical approaches in which AFM is coupled to other biophysical techniques, which can provide analytical information about lipid-protein interactions. At the end, a summary section will critically analyze the obtained results and will foresee the possible future developments that AFM will enable in the field of lipid-protein interactions.

\section{CHAPTER 1: SUPPORTED LIPID BILAYERS WITH RECONSTITUTED MEMBRANE PROTEINS}

Supported lipid bilayers consist of a lipid bilayer on a rigid substrate such as glass, silicon oxide or mica. They were initially developed by the McConnell's group to study the interaction of cells with lipid bilayers (McConnell et al., 1986; Castellana and Cremer, 2006). They can be assembled by two different strategies: the Langmuir Blodgett/Schaefer approach (Dufrêne et al., 1997) and the vesicle fusion technique (Brian and McConnell, 1984). The first technique is based on two consecutive transfers to a solid substrate of lipid monolayers assembled at the liquid/air interface in a Langmuir trough. The appealing feature of this approach is connected with the possibility of forming lipid bilayers characterized by a transbilayer lipid asymmetry, reproducing the actual situation of biological membranes. The vesicle fusion technique forms supported lipid bilayers from unilamellar vesicles in solution. Upon contact with surfaces, under specific conditions, unilamellar vesicles rupture, forming a planar bilayer. In both preparation strategies, the presence of a thin water layer between the bilayer leaflet nearer to the substrate (proximal leaflet) and the substrate itself allows to preserve lipid diffusion (Koenig et al., 1996). It is, however, to be stressed that the small thickness of the water layer might somehow limit the lipid diffusion with respect to the case in which a lipid leaflet is facing bulk water (see discussion below). The thin water layer allows also to host transmembrane proteins even in the case in which a small portion protrudes from the bilayer towards the support side. The vesicle fusion technique allows the incorporation of transmembrane proteins in the supported lipid bilayer more easily than the Langmuir-Blodgett/Schaefer one. Even if strategies for the incorporation of detergent solubilized transmembrane proteins in already formed supported lipid bilayers have been developed (Milhiet et al., 2006; Muller, 2006), the direct fusion of proteoliposomes on surfaces appears as a more practical approach. Moreover, the drawback of the technique, which exploits the addition of detergent to slightly destabilize the lipid bilayer in order to favor the insertion of solubilized membrane proteins, lies in the unknown amount of detergent which remains in the lipid bilayer. The residual detergent could affect both the thermodynamics of the lipid bilayer and the functionality of the proteins.

Vesicle fusion is usually performed starting from Small Unilamellar Vesicles (SUV) or Small Unilamellar Proteoliposomes, which means vesicles with a diameter of a few tens of nanometers. According to the most probable pathway for supported lipid bilayers formation from vesicles, the external face of the liposomes will face the solid support in the final bilayer configuration, while the internal layer will face the bulk of the solution. However, contradictory results have been reported about the formation of SLBs from vesicles containing transmembrane proteins (Contino et al., 1994; Salafsky et al., 1996). In some cases it has been found that proteins which exposed the active site to the bulk solution in vesicles also exposed the active site to the bulk solution in the SLB. In other cases, it has been found that a significant redistribution of protein orientation occurs during the SLB formation. Probably, the scenario at work depends on the specific case (Reimhult et al., 2009). Among the parameters to consider there are the size of the vesicles, their lipid composition, deposition temperature, and the nature of the support. The orientation of the proteins in the lipid bilayer can be connected to the orientation in the proteoliposomes, but the final structure on the surface depends on the rupture pathway of the vesicles and on possible reorientations of the proteins. The accurate orientation of the proteins in the SLB can be established by functional tests or by measuring the distribution of the height of the inclusions (Liu et al., 2009).

When supported lipid bilayers with reconstituted membrane proteins are studied by AFM, a homogeneous lipid to protein ratio in the vesicles is highly desired. This situation would allow more reproducible results and a uniform distribution of the proteins in the supported lipid bilayer in the case of a homogenous lipid phase (Vuong et al., 2010). In fact, the possibility that vesicles with different lipid to protein ratio have a different affinity for the solid substrate could make the obtained supported lipid bilayer with reconstituted membrane proteins largely independent from the real vesicle composition in the 
solution. Even if the control over the lipid to protein ratio in the vesicles is difficult, it is important to take into consideration this parameter when interpreting the obtained AFM images. It is usually found that the density of proteins in the supported lipid bilayers is lower than the nominal concentration used to prepare the proteoliposome sample (Seeger et al., 2009a). This behavior could be the result of the presence of lipid vesicles without proteins in solution, especially in the case of detergent removal by dialysis. Removal of detergent by other techniques, such as the use of Bio-Beads (Rigaud et al., 1997), offers the possibility of great improvements in the results both for the reconstitution of membrane proteins into vesicles and for the stripping of detergent from destabilized supported lipid bilayers.

The main drawback in the SLB model system stems from the small water gap between the lipid bilayer and the solid support (0.5-2 nm) (Bayerl and Bloom, 1990; Johnson et al., 1991; Koenig et al., 1996). This aspect could be a problem if membrane proteins with a large extramembraneous portion have to be incorporated in the bilayers. A contact between the proteins and the support could affect the protein conformation and, consequently, the function of the molecules. However, there are cases in which the reconstitution of membrane proteins in-plane on a solid substrate does not affect the protein functionality (Ataka et al., 2004). The interaction is strictly dependent on the nature of the substrate. On hydrophilic substrates such as mica, silicon oxide, and glass, the interactions which are established between the membrane and the substrate are sufficiently weak to prevent alterations of normal molecular behavior.

Lateral diffusion is an aspect to be considered when lipid-protein interactions are studied by exploiting supported lipid bilayers. Diffusion firstly regards lipids and then also proteins in the SLB. Recent experimental results have shown that the lipid diffusion in free standing bilayers (GUV, Giant Unilamellar Vesicles) is more than two times faster than in supported lipid bilayers measured in the same conditions (the diffusion coefficient is $D=7.8 \mu \mathrm{m}^{2} \mathrm{~s}^{-1}$ for GUVs and $D=3.1 \mu \mathrm{m}^{2} \mathrm{~s}^{-1}$ for SLBs) (Przybylo et al., 2006). A different and more complicated issue is the possible difference in diffusion coefficients between the two leaflets of the bilayer. Asymmetric dynamic properties involve the strength of the interleaflet coupling. In literature, different results can be found. Hetzer et al. (1998) found that the distal leaflet has a diffusion constant which is two times faster than the proximal leaflet, pointing to an independent behavior of the two leaflets. Recent results found the same translational diffusion coefficient for both leaflets within a $10 \%$ experimental uncertainty (Zhang and Granick, 2005). In the latter case a strong coupling between the two leaflets could be the reason for the same lateral mobility. We recently demonstrated that the interleaflet coupling is strongly related to the experimental details of the sample preparation, including preparation temperature and the type of support (Seeger et al., 2009b; Seeger et al., 2010). So, it is not always possible to compare the obtained results even if related to the same system.

Many membrane proteins perform their tasks by forming dynamic assemblies with other proteins in the membrane. The single molecule level study of the molecular interactions would increase our knowledge of biological processes. Dealing with the diffusion of membrane proteins in supported lipid bilayers, it is usually found that proteins are able to diffuse, but the diffusion coefficient is orders of magnitude lower than expected from proteins embedded in free standing bilayers (Müller et al., 2003). The reason for this behavior could be found in the thickness of the water layer between the membrane and the support which could increase frictional forces for membrane protein diffusion. Another explanation for the low diffusion coefficient is the presence of pinning points of the bilayer to the substrate which could produce an obstructed diffusion. Different types of motion, free diffusion, and obstructed diffusion, have indeed been observed by AFM on SLBs (Müller et al., 2003). It has been noted that the diffusion of membrane proteins is related to the fluidity of the lipid bilayer. Indeed, by increasing the temperature of the sample it is possible to observe an increase of the displacements of the proteins in the membrane, eventually reaching a situation in which the proteins are no more visualized by AFM (personal observations). A major limitation in the observation of protein diffusion by AFM is the low time resolution of the technique. This limit allows only the observation of slow dynamics. In particular, depending on the area imaged and the time interval between two consecutive images, limits in the determination of diffusion coefficients are encountered (Hughes et al., 2004; Casuso et al., 2009). For example, it has been hypothesized that the prevalent observation of membrane proteins in association with the more ordered regions of the lipid bilayer does not mean that the same proteins are not interacting with the more liquid phases, especially in the case of small proteins or peptides (Chiantia et al., 2006c). In fact, highly mobile components in the lipid bilayer might be not detected and a complementary technique should be used to exclude the presence of small mobile proteins in the fluid regions of the bilayer (see below). One of the most exciting area of development for the AFM technique in biological studies is the high-speed imaging which could allow the acquisition of time-lapse images with a very short time interval (see future trends) (Ando et al., 2007; Casuso et al., 2009). The reduced protein diffusion constant does not however imply an alteration of the functionality. For example, it has been demonstrated in a recent fluorescence study on the cooperativity of an ion-channel subunits performed on a solid SLB that, in spite of an apparent absence of mobility for all the molecules, only a small fraction showed no activity in response to a gating stimulus (Blunck et al., 2008). A method which is worthwhile being developed and exploited is that of assembling membranes on supports in which holes have been produced (Gonçalves et al., 2006). In this case it would be possible to image by AFM membrane proteins separating two aqueous compartments (see future trends), configuring thus a free standing membrane.

A further shortcoming of the use of supported lipid bilayers to study lipid-protein interaction is connected to the planar geometry of the model system. In many native membranes the geometry is curved and the curvature is usually induced by the presence of surface bound proteins or transmembrane proteins. Two-dimensional projection of the native 3D structure can be affected by artifacts in the apparent membrane organization (Olsen et al., 2008). In particular, the role of the membrane curvature in determining protein distribution or the role of proteins in influencing the curvature may be underestimated.

\section{CHAPTER 2: LIPID/PROTEIN INTERACTION STUDIED BY AFM}

The use of AFM on supported lipid bilayers containing proteins has provided a wealth of information on the distribution of membrane proteins relative to the lipid phase separation or on 
the effect of peptides and proteins on the structure of lipid bilayers. Since the introduction of the idea that domains could appear in biological membranes, it became evident that understanding the distribution of peptides and proteins in lipid bilayers would have been relevant for elucidating signaling pathways. Both theoretical (Gil et al., 1998) and experimental (Brown, 1994) efforts concentrated on the clustering and partitioning of proteins in lipid membranes. From an experimental point of view AFM represents one of the most suitable approaches due to its high lateral and vertical spatial resolution. The distribution of proteins and peptides along with the phase behavior of the lipid bilayer can be studied by AFM in a physiologic-like environment without the need of a labeling step. One of the first studies in this context concentrated on the distribution of peptides such as Gramicidin A, establishing the possibility for those peptides to form clusters in the lipid bilayer (Mou et al., 1996; Ivanova et al., 2003). In terms of membrane protein/lipid interactions, the distribution of either GPI-anchored proteins, lipidated proteins or transmembrane proteins in the presence of phase separation in lipid bilayers is particularly relevant for understanding trafficking processes of the membrane and the possible influence of phase separation on transmembrane protein function. This topic has been studied by AFM (Milhiet et al., 2002; Seeger et al., 2009b). In the following, we will concentrate on studies performed on these types of membrane proteins. We forward the readers interested in AFM studies on the interactions of small peptides, such as antimicrobial peptides, fusogenic peptides and cell-penetrating peptides with lipid bilayers to excellent reviews in the literature (Brasseur et al., 2008; El Kirat et al., 2010). The reader can also find interesting AFM works on the interaction of peripheral membrane proteins such as cytochroms with supported lipid bilayers (Choi and Dimitriadis, 2004; El Kirat and Morandat, 2009). It should be stressed that the examples we will show do not represent the general behavior of membrane proteins upon phase separation in the bilayer and that the results obtained by AFM should be as much as possible compared to the results coming from other biophysical techniques. The last requirement stems from the fact that the different model systems exploited could give different results.

Glycosylphosphatidylinositol (GPI)-anchored proteins (GPI-APs) are a class of proteins that are anchored to the membrane by means of a posttranslational lipid chain insertion (Mayor and Riezman, 2004). The lipid modification allows these proteins to be somehow related to the membrane trafficking mechanisms and to domain formation especially in the outer leaflet of the biological membrane. Understanding the mechanisms underlying the sorting of GPI-APs in a lipid bilayer is important in the context of the lateral heterogeneities in biological membranes and its functional role. One of the GPI-anchored proteins which has been studied by AFM is Placental Alkaline Phosphatase (PLAP) (Saslowsky et al., 2002; Giocondi et al., 2008). The aim of these studies was to establish whether PLAP was targeted to raft domains in lipid bilayers. To that aim, proteoliposomes with associated PLAP proteins were assembled and successively fused on a mica support or were inserted into preformed SLBs. The sample preparation technique assured the preservation of the protein functionality in the supported planar lipid bilayer and the proteins were mainly found associated with the most ordered domains of the lipid bilayer composed by synthetic mixture of SM and DOPC with and without cholesterol. The obtained results constitute a paradigmatic example useful to understand the relationship between different bilayer model systems. In fact, experiments performed on a similar lipid system and the same protein but on GUV, gave the striking result of the proteins mainly concentrated in the liquid disordered regions instead of the ordered ones (Kahya et al., 2005). The PLAP protein is considered an example of Detergent Resistant Membrane (DRM) associated protein. The finding that PLAP protein behaves differently in DRMs and in GUV can be explained on the basis of the alteration of the thermodynamics of the membrane when detergent is inserted and temperature changed to identify DRM areas. The behavior observed in AFM studies can also be affected by the interaction of the proteins with the support. In conclusion, this example highlights some of the problems connected with the identification of lipid rafts in biological membranes. In the case of GPI-anchored intestinal alkaline phosphatase (BIAP), the incorporation of the protein in an already formed SLB, demonstrated the preferential incorporation of the protein in the gel phase domains of different lipid mixtures (Giocondi et al., 2007a, 2008) (Figure 1). Also in this case, as in that of PLAP, the heterogeneity of the lateral membrane organization is able to influence the protein distribution. In the work on BIAP it was also possible to observe a transfer of lipids from the fluid to the gel phase or vice versa, upon protein insertion in the bilayer. This effect can be deduced by the variation of the relative area of the fluid and gel regions. The spontaneous insertion of these $\mathrm{GPI}$-anchored proteins into preformed lipid bilayers usually starts from the interface between different phase domains. This phenomenon can be related to the presence of both high line tension and
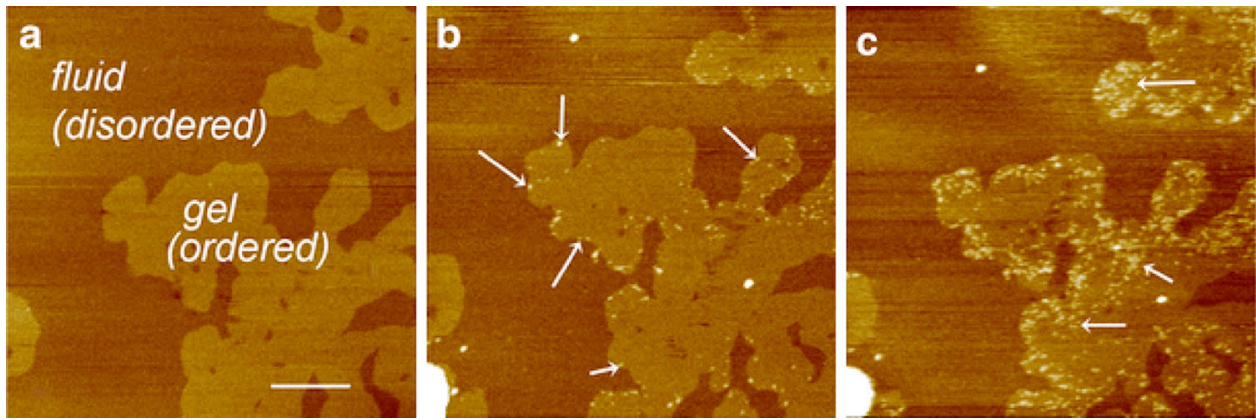

Figure 1. Sequence of AFM images showing the insertion of BIAP in DOPC/DPPC supported lipid bilayers. The insertion of the proteins was performed after the lipid bilayer was formed on the substrate. (a) Lipid bilayer showing the coexistence of fluid and gel domains before the addition of BIAP. (b) AFM image of the same area as in (a) 45' after the addition of BIAP. Arrows point to the BIAP proteins which insert preferentially at the boundary between fluid and gel domains. (c) After longer incubation time the proteins inserted also in the center of the gel domains (arrows). Scale bar $=1 \mu \mathrm{m}$. Reproduced with permission from (Giocondi et al., 2008). 
packing defects in this area. Considering the insertion and the redistribution of the lipids it is probable that GPI-anchored proteins, once in the lipid bilayer, recruit the most suitable lipid environment most likely by exploiting the hydrophobic matching interaction. The AFM can also be exploited to observe the redistribution of $\mathrm{GPI}$-anchored proteins after a temperature variation (Giocondi et al., 2007b). The obtained information can be fruitfully compared with results obtained from DRM extraction at low temperature.

In the case of other lipidated proteins, N-Ras proteins, it has been demonstrated by a combined fluorescence study on GUV and AFM study on supported lipid bilayers that the proteins behave in the same way in both model systems (Weise et al., 2010). In particular, lipidated N-Ras proteins have a strong preference for liquid disordered domains in the lipid bilayer (Nicolini et al., 2006). In a time-lapse AFM study on lipidated N-Ras proteins it has been demonstrated that the proteins preferentially partition in the liquid disordered phase and then migrate towards the domain boundaries where they decrease the domain line tension. (Weise et al., 2009). Moreover, N-Ras proteins form clusters which modify also the lipid distribution by creating lipid domains which did not exist before the insertion of the proteins.

The Atomic Force Microscope can also be exploited to study the partitioning of integral transmembrane proteins with respect to the lateral phase heterogeneity of the lipid bilayer. When integral membrane proteins are incorporated into lipid membranes and domain formation occurs, two effects result. The domain formation process appears to be modulated by the presence of the proteins according to the lipid protein interaction (Sperotto et al., 1989) and the domain structure influences the function of the proteins by introducing tensions which can act on the conformational changes of the proteins (Brown, 1994). The hydrophobic matching principle is usually evoked to explain the protein/lipid interactions and the distribution of the proteins in the bilayer relative to different phases which might exist. At the same time, if lipids of different hydrophobic thickness are present in the lipid bilayer, the protein can perform a lipid sorting at its interface on the basis of physical reasons, even in the absence of any chemical specificity. The hydrophobic thickness of the lipids strongly depends on the temperature of the system, especially near a phase transition region. Thus, it is reasonable to expect that the organization and the protein/lipid interactions change with temperature. A further partitioning effect comes from the existence of domain boundaries. It has been calculated that, for particular lipid compositions, when the system is in the phase transition region with a coexistence of gel and liquid domains, the proteins tend to be adsorbed at the phase boundaries with a region of fluid lipids around them (Dumas et al., 1997). This effect represent a sort of interfacial adsorption which is generally expected in a many-phase system with impurities that have no particular preference for any given phase and are therefore confined to the interface.

Seeger et al. (2009a) have studied the partitioning of the $\mathrm{K}^{+}$ channel KcsA (Doyle et al., 1998) in a POPE/POPG supported lipid bilayer when a phase transition of the lipid bilayer was induced. In that work the sample was prepared by the fusion of proteoliposomes on a mica surface and imaged by temperature controlled AFM. The obtained sample most likely reflects the vectorial incorporation of the KcsA molecules in the POPE/POPG liposomes, where the channels are incorporated almost exclusively in the outside-out configuration. This type of incorporation has been confirmed by a proteolytic assay (Cuello et al., 1998). The height distribution of the observed bumps points to a monomodal distribution. Considering the strong difference between the two extramembraneous portions of KcsA it is likely that the vectorial reconstitution is preserved also in the supported lipid bilayer. Figure 2 reports a sequence of AFM images of the lipid bilayer with the reconstituted proteins
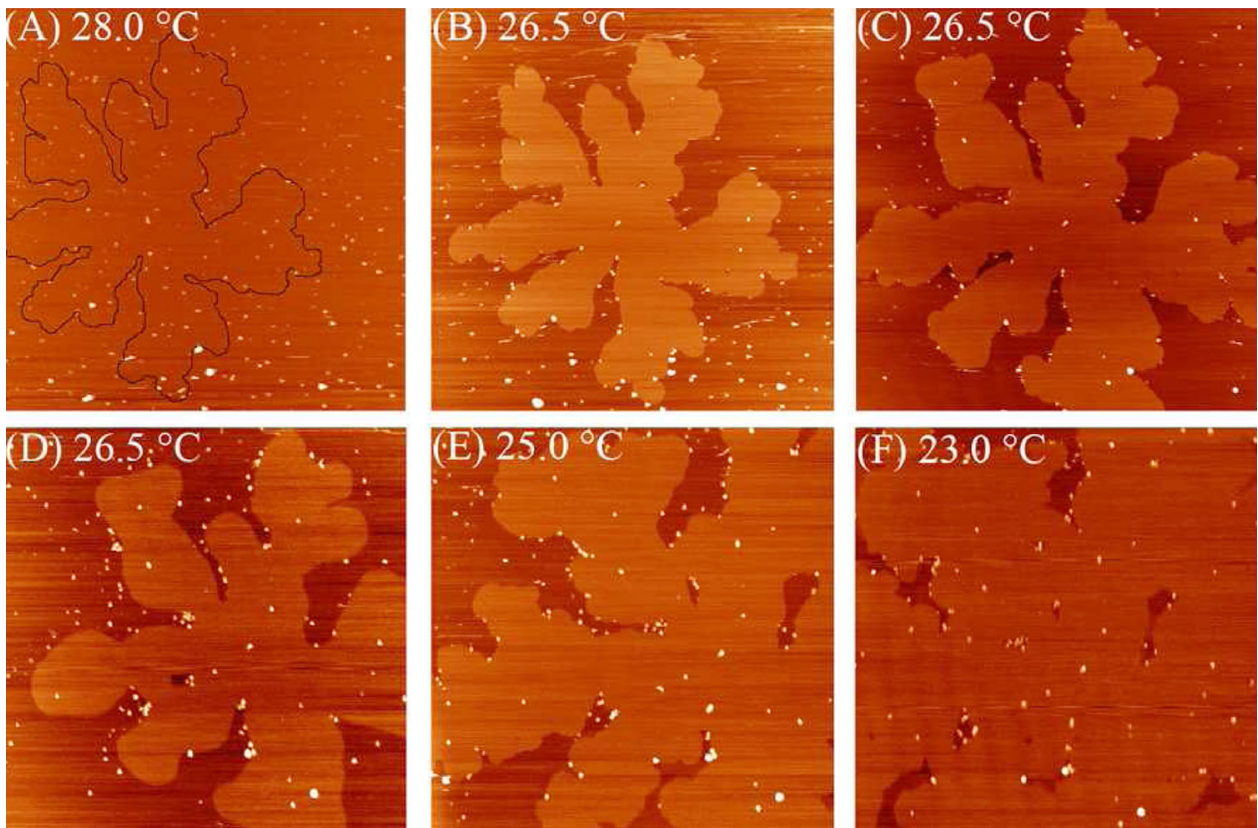

Figure 2. Series of AFM images $(10 \mu \mathrm{m} \times 10 \mu \mathrm{m})$ of the redistribution behavior of KcsA reconstituted in a SLB of POPE:POPG 3:1. (A) The SLB was equilibrated in the liquid disordered phase. The KcsA proteins are randomly distributed in the SLB. The black line is the outline of the solid ordered domain which formed in B. (B) A solid ordered domain (lighter area) was induced by cooling. The KcsA proteins were excluded from the solid ordered region. (C-D) The solid ordered domain was allowed to equilibrate. $(E-F)$ The phase transition and the protein redistribution proceeded upon further temperature decrease. 
obtained on the same sample area while the temperature was decreased. The observed bumps are ascribable to the cytoplasmic portion of KcsA facing the AFM tip. When the SLB with reconstituted $\mathrm{KcsA}$ molecules was imaged at $28^{\circ} \mathrm{C}$, the bilayer was in the liquid disordered state (Figure $2 A$ ) and the proteins were homogeneously distributed. Upon decreasing the temperature to $26.5^{\circ} \mathrm{C}$, the presence of a solid ordered lipid domain was induced (Figure 2B). The growing solid domain tended to exclude the proteins which remained mainly confined in the liquid disordered phase. Moreover, most of the proteins were found at the interface between the solid ordered and the liquid disordered domains. By further decreasing the temperature, the solid domain area increased and the proteins were mainly present in the decreasing liquid fraction of the bilayer (Figure $2 \mathrm{C}$ ) and were eventually induced to form clusters. The observed behavior of the $\mathrm{KcsA}$ protein can be rationalized on the basis of the hydrophobic matching mechanism between the hydrophobic thickness of the protein and of the lipid bilayer. The variation of the hydrophobic thickness of the lipid membrane occurring at the transition between the liquid disordered and solid ordered phases can induce a strong redistribution of the proteins in the membrane. The confinement of many proteins at the domain boundaries can be explained by the above introduced concept of interfacial adsorption. Figure 3 shows what happens to the same lipid/ protein (POPE/POPG plus KcsA) system when the temperature is increased starting from a value which assures the coexistence of gel and liquid phases. In this case the gel domains recede and some of the proteins follow the domain boundary, suggesting the presence of an attractive potential for the inclusions towards the interfacial region. Some of the proteins seem to be immobile in the AFM time-lapse images. It is probable that some of them are pinned to the substrate. It has been shown that when a supported lipid bilayer with reconstituted KcsA proteins is obtained, only a small fraction of proteins is inactive (Blunck et al., 2008). The inactive proteins could correspond to the immobile proteins observed in the AFM images.

The functional activity of membrane proteins can be strongly affected by their partitioning in different domains, especially in the case where function is coupled to a conformational change of the protein. This phenomenon has been demonstrated by Brown (1994) in the case of rhodopsin but it is probably the reason for the variation in the functional activity of proteins in the phase coexistence region observed for KcsA (personal observations) and for the sarcoplasmic reticulum $\mathrm{Ca}^{2+}$ release channel (Cannon et al., 2003). In the work by Cannon et al., the altered protein functionality registered at the lipid/protein phase coexistence region of the gel/liquid transition was attributed to the preference of the proteins for the domain boundaries. This preference has been ascribed to the presence of an attractive potential towards the domain interface. The localization of the proteins at the domain boundaries can also be a method to decrease the line tension which arises due to the different thickness of the two different domains and the hydrophobic character of the inner region of the lipid bilayer. This behavior is evident in the sequence of AFM images relative to a POPE/POPG bilayer with reconstituted KcsA molecules obtained at increasing temperature (Figure 3). Another consequence of protein segregation is the increased possibility of cluster formation which might affect their behavior (Molina et al., 2006). Variation of the lipid material properties across the main phase transition can explain a modification of the functionality of transmembrane proteins. It has already been reported that the Arrhenius plots of the functional activity of many membrane bound enzymes or transport membrane proteins show breaks which are considered hallmarks of lipid phase transitions (Silvius and McElhaney, 1980). For example, in the phase transition region, in which lipid compressibility is high, fluctuations in enthalpy are strong and the relaxation times of lipids after a pressure perturbation are expected to be low (Grabitz et al., 2002). In particular, strong fluctuations are localized at the domain boundaries. The presence of strong fluctuations in the lipid bilayer could influence the function of membrane proteins, especially if their function implies large conformational variations at the protein/ lipid interface.

Another consequence of protein partitioning in lipid domains is the modification of the lipid environment around the proteins. In the case of KcsA in lipid bilayers composed of a mixture of POPE/POPG 3:1, it is expected that in the phase coexistence regions the liquid domains are particularly enriched in POPG due to its low melting temperature. The increased POPG concentration in the lipid bilayer felt by the proteins which are confined in the liquid domains could modify the protein behavior if specific lipid/protein interactions are at work.

All these expected behaviors have been observed in functional studies of KcsA in Black Lipid Membrane (BLM) model system (personal observations). In particular, at the single molecule level, it has been observed that all the functional features of KcsA, open probability, single molecule conductance, and open and closed dwell times, show a biphasic behavior when the temperature is decreased with the inversion point near to the starting of the lipid


Figure 3. Redistribution of KcsA upon domain melting. (A) A KcsA protein (in the red ellipse) is aligned at the domain boundaries between the solid ordered and liquid disordered phase. (B) Upon heating the solid ordered domain melts and hence occupies a smaller area fraction. The protein stays aligned at the solid ordered/liquid disordered interface (C-D). The redistribution behavior of two KcSA proteins were followed in another area of the SLB and at other temperatures. 
phase transition. According to what has been observed by AFM, the functional behavior can in this case be related to partitioning of the proteins in the different lipid phases or at the domain boundaries.

\section{CHAPTER 3: COUPLING AFM WITH OTHER TECHNIQUES}

One of the main limitations of AFM is its lack of chemical information. Most often, AFM provides only morphological information to recognize specific components in the sample. Even in the case of lipid domain formation, AFM offers details on the topography of the lipid bilayer to recognize the different phases. Other techniques which are able to complement these data by other details such as order parameters or lipid dynamics are therefore welcome (Chiantia et al., 2006b; Oreopoulos and Yip, 2009). In general, optical techniques are used to complement AFM data. Optical information usually requires the use of fluorescent labels to be obtained. Moreover, optical techniques could provide higher time resolution than AFM. By complementing AFM and optical techniques a more in depth view of lipid/ protein interactions can be obtained. Different optical techniques have been combined with AFM. Among these techniques there are fluorescence microscopy (Frankel et al., 2006), confocal fluorescence microscopy (Shaw et al., 2006), (polarized) total internal reflection fluorescence microscopy (Slade et al., 2006; Oreopoulos and Yip, 2009), fluorescence correlation spectroscopy (Burns et al., 2005; Chiantia et al., 2006a) and attenuated total reflection-Fourier transform infrared (ATR-FTIR) spectroscopy (Verity et al., 2009).

A combined approach of Atomic Force Microscopy, fluorescence imaging and Fluorescence Correlation Spectroscopy was exploited to study the partitioning of membrane proteins in ceramide-rich domains (Chiantia et al., 2006b). Ceramide-rich domains are highly ordered gel domains which may have a role in sorting of membrane proteins. GPI-anchored proteins whose behavior is characterized by a strong partitioning inside liquid-ordered domains, such as GPI-PLAP, showed a strong partitioning also in Ceramide-rich domains. Figure 4A shows the presence of different lipid phases on the same bilayer: liquid disordered, liquid ordered and ceramide domains, from the lowest to the highest one. The presence of GPI-PLAP proteins is evidenced by the white bumps. Figure $4 B$ shows a Laser Scanning Microscopy image of the same sample used for figure $4 \mathrm{~A}$. The green channel is representative of the fluorescent lipid enriched in the liquid disordered phase whereas the liquid ordered phase is represented by the darker regions. The red circles highlight the ceramide-rich domains previously identified by AFM imaging on the same sample area. The red channel identifies the presence of fluorescently labeled GPI-PLAP proteins which are not excluded from ceramide-rich domains. A quantitative measurement of the partitioning of the proteins in the different lipid phases can be provided by Fluorescence Correlation Spectroscopy.

Another interesting hybrid technique (AFM-ATR-FTIR) has been recently applied to study protein/lipid interaction (Verity et al., 2009). The use of Infrared Spectroscopy consents to avoid the use of fluorescent labels which may alter the thermodynamics of the protein/lipid ensemble. At the same time, FTIR in the ATR configuration allows to study the conformation of proteins in the lipid bilayer and, if used with linearly polarized illumination it also allows to obtain the orientation of the secondary elements of the


Figure 4. (A) AFM image showing a SM/DOPC/ceramide/cholesterol supported lipid bilayer with reconstituted GPI-PLAP in which different phases are present. From the darkest to the lightest the phases are: liquid disordered, liquid ordered and ceramide-enriched. Protein cluster/aggregates are represented by the white bumps. (B) Laser Scanning Microscopy fluorescence image of the same sample as in A. The green channel identifies the liquid disordered phase whereas the red channel identifies the PLAP protein. The red circles refer to ceramide-rich regions previously recognized by parallel AFM imaging. The black arrow represents the scanning path used by FCS to collect the signal from the proteins in each phase. Scale bar $=2 \mu \mathrm{m}$. Reproduced with permission from (Chiantia et al., 2006b).

proteins relative to the plane of the membrane. With this set-up, even if the spectroscopic technique does not have lateral space resolution, it is possible to correlate the partitioning of the proteins in the lipid bilayer to their possible conformational changes.

Another analytical technique which has been usefully coupled to AFM imaging is High Resolution Secondary lon Mass Spectrometry (Nano SIMS) (Kraft et al., 2006). In this case it has been possible to perform a chemical analysis on lipid domains demonstrating the lipid compositional heterogeneity in a freeze-dried phase separated lipid bilayer composed by DLPC/ DSPC with a lateral resolution of approximately 100 nanometers.

\section{CONCLUSIONS AND FUTURE TRENDS}

The high lateral and vertical resolution of AFM and the possibility to work under near physiological conditions make AFM a 
technique of choice to study the interactions between lipids and proteins. In particular, the partitioning of membrane associated proteins can be studied as a function of lipid composition, phase state of the bilayer and temperature. Spectroscopic information on lipid/protein interactions can be retrieved by coupling of AFM imaging with more analytical techniques. Much of the studies on this topic have been performed till now on model systems. Some experiments on native membranes have been performed and it is likely that in the future the attention will be focused on more and more complex systems (Alessandrini et al., 2008).

A combination of theoretical and experimental studies will allow a better understanding of the interaction of SLBs with the support in order to control the behavior of the lipid bilayer and to reproduce situations very similar to those experienced by the plasma membrane. New approaches such as SLBs on nano-holes (Gonçalves et al., 2006; Steltenkamp et al., 2006) will allow a better understanding of the bilayer/substrate interaction and will permit to expose the membrane to different water phases at the two sides. For examples, the possibility to fill the holes with a gel material similar to the cell cytoplasm could reproduce a situation very similar to the biological cell case.

New advancements in the elucidation of lipid/protein interaction exploiting AFM are surely connected to technical improvements. Among these improvements, the increase in time resolution of AFM imaging is a central issue. A great effort is being dedicated to this technical aspect and it is now possible to image dynamic processes in liquid with a time resolution of less than 1 second per image (Ando et al., 2008; Shibata et al., 2010). In the context of lipid/protein interaction the high time resolution could provide useful information about dynamic processes such as lipid domain formation and protein diffusion.

At the same time, progresses in the coupling of AFM with other signals such as fluorescence and Raman spectroscopy will increase our knowledge of lipid/protein interaction.

AFM operated in an electrochemical cell has already shown to be able to provide intriguing evidences in the response of a cell membrane bearing exogenous ion channels to a variation in the transmembrane potential such to elicit channel gating (Alessandrini et al., 2008). Similarly, it is also to be hoped that the functional activity of membrane proteins such as ion channels will be probed by a conductive AFM tip, suitable for liquid operation, along with the topographical information. In this fascinating situation it will be possible to directly correlate protein partitioning with the modification of their activity. In this context the activity of AFM cantilevers and tips modification in order to make them able to measure ion currents in solution already started (Menozzi et al., 2005; Gullo et al., 2006).

AFM applied in the force spectroscopy mode to membrane proteins will also increase our knowledge about the intra and inter-molecular interactions of these proteins which are relevant to drive protein folding, oligomerization and functional activity (Janovjak et al., 2008).

\section{REFERENCES}

Alessandrini A, Gavazzo P, Picco C, Facci P. 2008. Voltage-induced morphological modifications in oocyte membranes containing exogenous $\mathrm{K}^{+}$ channels studied by electrochemical scanning force microscopy. Microsc. Res. Tech. 71: 274-278.

Ando T, Uchihashi T, Fukuma T. 2008. High-speed atomic force microscopy for nano-visualization of dynamic biomolecular processes. Prog. Surf. Sci. 83: 337-437.

Ando T, Uchihashi T, Kodera N, Yamamoto D, Taniguchi M, Miyagi A, Yamashita H. 2007. High-speed atomic force microscopy for observing dynamic biomolecular processes. J. Mol. Recognit. 20: 448-458.

Ataka K, Giess F, Knoll W, Naumann R, Haber-Pohlmeier S, Richter B, Heberle J. 2004. Oriented attachment and membrane reconstitution of His-tagged cytochrome c oxidase to a gold electrode: in situ monitoring by surface-enhanced infrared absorption spectroscopy. J. Am. Chem. Soc. 126: 16199-16206.

Bayerl TM, Bloom M. 1990. Physical properties of single phospholipid bilayers adsorbed to micro glass beads. A new vesicular model system studied by $2 \mathrm{H}$-nuclear magnetic resonance. Biophys. J. 58: 357-362.

Blunck R, McGuire H, Hyde HC, Bezanilla F. 2008. Fluorescence detection of the movement of single KcsA subunits reveals cooperativity. Proc. Natl Acad. Sci. USA 105: 20263-20268.

Brasseur R, Deleu M, Mingeot-Leclercq MP, Francius G, Dufrene YF. 2008. Probing peptide-membrane interactions using AFM. Surf. Interface Anal. 40: $151-156$

Brian AA, McConnell HM. 1984. Allogeneic stimulation of cytotoxic T cells by supported planar membranes. Proc. Natl Acad. Sci. USA 81: 6159-6163.

Brown MF. 1994. Modulation of rhodopsin function by properties of the membrane bilayer. Chem. Phys. Lipid. 73: 159-180.

Burns AR, Frankel DJ, Buranda T. 2005. Local mobility in lipid domains of supported bilayers characterized by atomic force microscopy and fluorescence correlation spectroscopy. Biophys. J. 89: 1081-1093.

Cannon B, Hermansson M, Gyorke S, Somerharju P, Virtanen JA, Cheng KH. 2003. Regulation of calcium channel activity by lipid domain formation in planar lipid bilayers. Biophys. J. 85: 933-942.
Castellana ET, Cremer PS. 2006. Solid supported lipid bilayers: from biophysical studies to sensor design. Surf. Sci. Rep. 61: 429-444.

Casuso I, Kodera N, Le Grimellec C, Ando T, Scheuring S. 2009. Contactmode high-resolution high-speed atomic force microscopy movies of the purple membrane. Biophys. J. 97: 1354-1361.

Chiantia S, Kahya N, Ries J, Schwille P. 2006a. Effects of ceramide on liquid-ordered domains investigated by simultaneous AFM and FCS. Biophys. J. 90: 4500-4508.

Chiantia S, Ries J, Chwastek G, Carrer D, Li Z, Bittman R, Schwille P. 2006b. Role of ceramide in membrane protein organization investigated by combined AFM and FCS. Biochim. Biophys. Acta 1778: 1356-1364.

Chiantia S, Ries J, Kahya N, Schwille P. 2006c. Combined AFM and two-focus SFCS study of raft-exhibiting model membranes. Chem. Phys. Chem. 7: 2409-2418.

Choi EJ, Dimitriadis EK. 2004. Cytochrome $c$ adsorption to supported, anionic lipid bilayers studied via atomic force microscopy. Biophys. J. 87: 3234-3241.

Contino PB, Hasselbacher CA, Ross JB, Nemerson Y. 1994. Use of an oriented transmembrane protein to probe the assembly of a supported phospholipid bilayer. Biophys. J. 67: 1113-1116.

Cuello LG, Romero JG, Cortes DM, Perozo E. 1998. pH-dependent gating in the Streptomyces lividans K+ channel. Biochemistry 37: 3229-3236.

Dufrêne YF, Barger WR, Green JBD, Lee GU. 1997. Nanometer-scale surface properties of mixed phospholipid monolayers and bilayers. Langmuir 13: $4779-4784$

Dumas F, Sperotto MM, Lebrun M-C, Tocanne J-F, Mouritsen OG. 1997. Molecular sorting of lipids by bacteriorhodopsin in dilauroylphosphatidylcholine/distearoylphosphatidylcholine lipid bilayers. Biophys. J. 73: 1940-1953.

Doyle DA, Morais Cabral J, Pfuetzner RA, Kuo A, Gulbis JM, Cohen SL, Chait BT, Mackinnon R. 1998. The structure of the potassium channel: molecular basis of $\mathrm{K}^{+}$conduction and selectivity. Science 280: 69-77.

Eggeling C, Ringemann C, Medda R, Schwarzmann G, Sandhoff K, Polyakova S, Belov VN, Hein B, von Middendorff C, Schönle A, Hell SW. 2009. Direct observation of the nanoscale dynamics of membrane lipids in a living cell. Nature 457: 1159-1162. 
El Kirat K, Morandat S., 2009. Cytochrome $c$ interaction with neutral lipid membranes: influence of lipid packing and protein charges. Chem. Phys. Lipid. 162: 17-24.

El Kirat K, Morandat S, Dufrêne YF. 2010. Nanoscale analysis of supported lipid bilayers using atomic force microscopy. Biochim. Biophys. Acta 1798: $750-765$.

Engel A, Gaub HE. 2008. Structure and mechanics of membrane proteins. Annu. Rev. Biochem. 77: 127-148.

Engel A, Müller DJ. 2000. Observing single biomolecules at work with the atomic force microscope. Nat. Struct. Biol. 7: 715-718.

Engelman DM. 2005. Membranes are more mosaic than fluid. Nature 438: 378-580.

Frankel DJ, Pfeiffer JR, Surviladze Z, Johnson AE, Oliver JM. 2006. Revealing the topography of cellular membrane domains by combined atomic force microscopy/fluorescence imaging. Biophys. J. 90: 2404-2413.

Gil T, Ipsen JH, Mouritsen OG, Sabra MC, Sperotto MM, Zuckermann MJ. 1998. Theoretical analysis of protein organization in lipid membranes. Biochim. Biophys. Acta 1376: 245-266.

Giocondi MC, Besson F, Dosset P, Milhiet PE, Le Grimellec C. 2007 a. Remodeling of ordered membrane domains by GPI-anchored intestinal alkaline phosphatase. Langmuir 23: 9358-9364.

Giocondi MC, Besson F, Dosset P, Milhiet PE, Le Grimellec C. 2007b. Temperature-dependent localization of GPI-anchored intestinal alkaline phosphatase in model rafts. J. Mol. Recognit. 20: 531537.

Giocondi MC, Seantier B, Dosset P, Milhiet PE, Le Grimellec C. 2008. Characterizing the interactions between GPI-anchored alkaline phosphatases and membrane domains by AFM. Pflugers Arch. 456: 179-188.

Gonçalves RP, Agnus G, Sens P, Houssin C, Bartenlian B, Scheuring S. 2006. Two-chamber AFM: probing membrane proteins separating two aqueous compartments. Nat. Method. 12: 1007-1012.

Grabitz P, Ivanova VP, Heimburg T. 2002. Relaxation kinetics of lipid membranes and its relation to the heat capacity. Biophys. J. 82: 299-309.

Gullo MR, Frederix PL, Akiyama T, Engel A, deRooij NF, Staufer U. 2006. Characterization of microfabricated probes for combined atomic force and high-resolution scanning electrochemical microscopy. Anal. Chem. 78: 5436-5442.

Hetzer M, Heinz S, Grage S, Bayerl TM. 1998. Asymmetric molecular friction in supported phospholipid bilayers revealed by NMR measurements of lipid diffusion. Langmuir 14: 982-984.

Hughes T, Strongin B, Gao FP, Vijayvergiya V, Busath DD, Davis RC. 2004 AFM visualization of mobile influenza A M2 molecules in planar bilayers. Biophys. J. 87: 311-322.

Ivanova VP, Makarov IM, Schäffer TE, Heimburg T. 2003. Analyzing heat capacity profiles of peptide-containing membranes: cluster formation of gramicidin A. Biophys. J. 84: 2427-2439.

Jacobson K, Mouritsen OG, Anderson RG. 2007. Lipid rafts: at a crossroad between cell biology and physics. Nat. Cell Biol. 9: 7-14.

Janovjak H, Sapra KT, Kedrov A, Müller DJ. 2008. From valleys to ridges: exploring the dynamic energy landscape of single membrane proteins. Chem. Phys. Chem. 9: 954-966.

Jensen MØ, Mouritsen OG. 2004. Lipids do influence protein function-the hydrophobic matching hypothesis revisited. Biochim. Biophys. Acta 1666: 205-226.

Johnson SJ, Bayerl TM, McDermott DC, Adam GW, Rennie AR, Thomas RK, Sackmann E. 1991. Structure of an adsorbed dimyristoylphosphatidylcholine bilayer measured with specular reflection of neutrons. Biophys. J. 59: 289-294.

Kahya N, Brown DA, Schwille P. 2005. Raft partitioning and dynamic behavior of human placental alkaline phosphatase in giant unilamellar vesicles. Biochemistry 44: 7479-7489.

Koenig BW, Kruger S, Orts WJ, Majkrzak CF, Berk NF, Silverton JV, Gawrisch K. 1996. Neutron reflectivity and atomic force microscopy studies of a lipid bilayer in water adsorbed to the surface of a silicon single crystal. Langmuir 12: 1343-1350.

Kraft ML, Weber PK, Longo ML, Hutcheon ID, Boxer SG. 2006. Phase separation of lipid membranes analyzed with high-resolution secondary ion mass spectrometry. Science 313: 1948-1951.

Lee AG. 2005. How lipids and proteins interact in a membrane: a molecular approach. Mol. Biosyst. 3: 203-212.

Lee AG. 2009. The effects of lipids on channel function. J. Biol. 8: 86.

Lingwood D, Simons K. 2010. Lipid rafts as a membrane-organizing principle. Science 327: 46-50.
Liu TH, Hsu SH, Huang YT, Lin SM, Huang TW, Chuang TH, Fan SK, Fu CC, Tseng FG, Pan RL. 2009. The proximity between C-termini of dimeric vacuolar $\mathrm{H}^{+}$-pyrophosphatase determined using atomic force microscopy and a gold nanoparticle technique. FEBS J. 276: 4381-4394.

Marius P, Zagnoni M, Sandison ME, East JM, Morgan H, Lee AG. 2008. Binding of anionic lipids to at least three nonannular sites on the potassium channel KcsA is required for channel opening. Biophys. J. 94: 1689-1698.

Mayor S, Riezman H. 2004. Sorting GPI-anchored proteins. Nat. Rev. Mol. Cell Biol. 2: 110-120.

McConnell HM, Watts TH, Weis RM, Brian AA. 1986. Supported planar membranes in studies of cell-cell recognition. Biochim. Biophys. Acta 864: $95-106$.

Menozzi C, Carlo Gazzadi G, Alessandrini A, Facci P. 2005. Focused ion beam-nanomachined probes for improved electric force microscopy. Ultramicroscopy 104: 220-225.

Milhiet PE, Gubellini F, Berquand A, Dosset P, Rigaud JL, Le Grimellec C, Lévy D. 2006. High-resolution AFM of membrane proteins directly incorporated at high density in planar lipid bilayer. Biophys. J. 91: 3268-3275.

Milhiet PE, Giocondi MC, Baghdadi O, Ronzon F, Roux B, Le Grimellec C. 2002. Spontaneous insertion and partitioning of alkaline phosphatase into model lipid rafts. EMBO Rep. 3: 485-490.

Mingeot-Leclercq MP, Deleu M, Brasseur R, Dufrêne YF. 2008. Atomic force microscopy of supported lipid bilayers. Nat. Protoc. 3: 1654-1659.

Molina ML, Barrera FN, Fernández AM, Poveda JA, Renart ML, Encinar JA, Riquelme G, González-Ros JM. 2006. Clustering and coupled gating modulate the activity in KcsA, a potassium channel model. J. Biol. Chem. 281: 18837-18848.

Mou J, Czajkowsky DM, Shao Z. 1996. Gramicidin A aggregation in supported gel state phosphatidylcholine bilayers. Biochemistry $\mathbf{3 5}$ : 3222-3226.

Muller DJ. 2006. Out and in: simplifying membrane protein studies by AFM. Biophys. J. 91: 3133-3134.

Müller DJ. 2008. AFM: a nanotool in membrane biology. Biochemistry 47: 7986-7998.

Müller DJ, Engel A. 2007. Atomic force microscopy and spectroscopy of native membrane proteins. Nat. Protocol. 2: 2191-2197.

Müller DJ, Engel A, Matthey U, Meier T, Dimroth P, Suda K. 2003. Observing membrane protein diffusion at subnanometer resolution. J. Mol. Biol. 327: 925-930.

Müller DJ, Janovjak H, Lehto T, Kuerschner L, Anderson K. 2002. Observing structure, function and assembly of single proteins by AFM. Prog. Biophys. Mol. Biol. 79: 1-43.

Nicolini C, Baranski J, Schlummer S, Palomo J, Lumbierres-Burgues $M$, Kahms M, Kuhlmann J, Sanchez S, Gratton E, Waldmann H, Winter R. 2006. Visualizing association of N-Ras in lipid microdomains: influence of domain structure and interfacial adsorption. J. Am. Chem. Soc. 128: 192-201.

Olsen JD, Tucker JD, Timney JA, Qian P, Vassilev C, Hunter CN. 2008. The organization of LH2 complexes in membranes from Rhodobacter sphaeroides. J. Biol. Chem. 283: 30772-30779.

Oreopoulos J, Yip CM. 2009. Probing membrane order and topography in supported lipid bilayers by combined polarized total internal reflection fluorescence-atomic force microscopy. Biophys. J. 96: 1970-1984.

Przybylo M, Sýkora J, Humpolícková J, Benda A, Zan A, Hof M. 2006. Lipid diffusion in giant unilamellar vesicles is more than 2 times faster than in supported phospholipid bilayers under identical conditions. Langmuir 22: 9096-9099.

Reimhult E, Kasemo B, Höök F. 2009. Rupture pathway of phosphatidylcholine liposomes on silicon dioxide. Int. J. Mol. Sci. 10: 1683-1696.

Rigaud JL, Mosser G, Lacapere JJ, Olofsson A, Levy D, Ranck JL. 1997. Bio-Beads: an efficient strategy for two-dimensional crystallization of membrane proteins. J. Struct. Biol. 118: 226-235.

Sackmann E. 1984. Physical basis for trigger processes and membrane structures. In Biological Membranes, Chapman D (ed.). Academic Press: London; 105-143.

Salafsky J, Groves JT, Boxer SG. 1996. Architecture and function of membrane proteins in planar supported bilayers: A study with photosynthetic reaction centers. Biochemistry 35: 14773-14781.

Saslowsky DE, Lawrence J, Ren X, Brown DA, Henderson RM, Edwardson JM. 2002. Placental alkaline phosphatase is efficiently targeted to rafts in supported lipid bilayers. J. Biol. Chem. 277: 26966-26970.

Schmidt D, Jiang QX, MacKinnon R. 2006. Phospholipids and the origin of cationic gating charges in voltage sensors. Nature 444: 775-779. 
Seeger HM, Bortolotti CA, Alessandrini A, Facci P. 2009a. Phasetransition-induced protein redistribution in lipid bilayers. J. Phys. Chem. B 113: 16654-16659.

Seeger HM, Di Cerbo A, Alessandrini A, Facci P. 2010. Supported lipid bilayers on mica and silicon oxide: comparison of the main phase transition behavior. J. Phys. Chem. B 114: 8926-8933.

Seeger HM, Marino G, Alessandrini A, Facci P. 2009b. Effect of physical parameters on the main phase transition of supported lipid bilayers. Biophys. J. 97: 1067-1076.

Shaw JE, Epand RF, Sinnathamby K, Li Z, Bittman R, Epand RM, Yip CM. 2006. Tracking peptide-membrane interactions: insights from in situ coupled confocal-atomic force microscopy imaging of NAP-22 peptide insertion and assembly. J. Struct. Biol. 155: 458-469.

Shibata M, Yamashita H, Uchihashi T, Kandori H, Ando T. 2010. Highspeed atomic force microscopy shows dynamic molecular processes in photo-activated bacteriorhodopsin. Nat. Nanotech. 5: 208212.

Silvius JR, McElhaney RN. 1980. Membrane lipid physical state and modulation of the $\mathrm{Na}^{+}, \mathrm{Mg}^{2+}$-ATPase activity in Acholeplasma laidlawii B. Proc. Natl Acad. Sci. USA 77: 1255-1259.

Simons K, Ikonen E. 1997. Functional rafts in cell membranes. Nature 387: 569-572.

Singer SJ, Nicholson GL. 1972. The fluid mosaic model of the structure of cell membranes. Science 175: 720-731.
Slade AL, Schoeniger JS, Sasaki DY, Yip CM. 2006. In situ scanning probe microscopy studies of tetanus toxin-membrane interactions. Biophys. J. 91: 4565-4574.

Sperotto MM, Ipsen JH, Mouritsen OG. 1989. Theory of protein-induced lateral phase separation in lipid membranes. Cell Biophys. 14: 79-95.

Steltenkamp S, Muller MM, Deserno M, Hennesthal C, Steinem C, Janshoff A. 2006. Mechanical properties of pore-spanning lipid bilayers probed by atomic force microscopy. Biophys. J. 91: 217-226.

Verity JE, Chhabra N, Sinnathamby K, Yip CM. 2009. Tracking molecular interactions in membranes by simultaneous ATR-FTIR-AFM. Biophys. J. 97: 1225-1231.

Vuong N, Baenziger JE, Johnston LJ. 2010. Preparation of reconstituted acetylcholine receptor membranes suitable for AFM imaging of lipid-protein interactions. Chem. Phys. Lipid. 163: 117-126.

Weise K, Triola G, Brunsveld L, Waldmann H, Winter R. 2009. Influence of the lipidation motif on the partitioning and association of N-Ras in model membrane subdomains. J. Am. Chem. Soc. 131: 1557-1564.

Weise K, Triola G, Janosch S, Waldmann H, Winter R. 2010. Visualizing association of lipidated signaling proteins in heterogeneous membranes-Partitioning into subdomains, lipid sorting, interfacial adsorption, and protein association. Biochim. Biophys. Acta 1798: 14091417.

Zhang L, Granick S. 2005. Lipid diffusion in outer and inner leaflets of planar supported bilayers. J. Chem. Phys. 123: 211104-211107. 